\title{
El Trabajo Fin de Grado en el Grado de Criminología: una herramienta de integración de competencias para los estudiantes y una experiencia de trabajo coordinado para los profesores ${ }^{1}$
}

\author{
Ana Isabel Cerezo Domíngueza ${ }^{a}$, María José Benítez Jiménez y Lorea Arenas García ${ }^{\mathrm{c}}$ \\ aProfesora Titular de Derecho penal y Criminología de la Universidad de Málaga \\ (aicerezo@uma.es), bAyudante doctor de Derecho penal y Criminología de la Universidad \\ de Málaga (mjbenitez@uma.es) y ${ }^{\mathrm{c}}$ Docente investigadora en formación de la Universidad \\ de Málaga (lorea@uma.es).
}

\begin{abstract}
In the Faculty of Law at the University of Malaga we have created an educational tool that aims students to achive an optimal level in developing the final degree work, compulsory subject in the last year of the Criminology studies. To do this, teachers will be working with students fron the first year all the necessary skills to achive this objective. In a coordinated way, by semester and year, each teacher will perform with students certain educational activities that allow the acquisition of skills such as improving written expression, proper management of ICT, the correct use of quotations literature, developing group work and oral expression, among others.
\end{abstract}

Keywords: final degree work, educational skills, coordination.

\begin{abstract}
Resumen: En la Facultad de derecho de la Universidad de Málaga hemos creado una herramienta docente que persigue que los alumnos alcancen un nivel óptimo en la elaboración del trabajo fin de grado, asignatura obligatoria del último curso del Grado de Criminología. Para ello, los profesores van a ir trabajando con los alumnos, desde el primer curso, todas las competencias necesarias para alcanzar este objetivo. De forma coordinada por curso y semestre, cada profesor realizará con los alumnos determinadas actividades docentes que permitan la adquisición de competencias tales como la mejorar la expresión escrita, el manejo adecuado de las tecnologías de la información y de la comunicación, la utilización correcta de citas bibliográficas, la elaboración de trabajos en grupo así como la expresión oral, entre otras.
\end{abstract}

Palabras clave: trabajo fin de grado, competencias, coordinación.

\footnotetext{
${ }^{1}$ El presente trabajo forma parte del Proyecto de Innovación Educativa denominado "El Trabajo Fin de Grado en la Facultad de Derecho: una herramienta de integración de competencias para los estudiantes y una experiencia de trabajo coordinado para los profesores", PIE 15-75. Entidad financiadora: Universidad de Málaga. Duración 01/01/2015 a 01/01/2017 (24 MESES) Investigadora principal: CARMEN MARÍA ÁVILA RODRÍGUEZ. Número de investigadores participantes: 41.
} 


\section{Introducción}

El art. 2 de la Ley Orgánica 6/2001, de 21 de diciembre, de Universidades (en adelante, LOU) afirma en su apartado $1^{\circ}$ la autonomía de las Universidades (las Universidades ... desarrollan sus funciones en régimen de autonomía), precisando en su apartado $2^{\circ}$ el elenco de facultades o competencias en las cuales aquélla se expresa ${ }^{2}$ Pues bien, de acuerdo con el citado precepto la autonomía de las Universidades comprende "la elaboración y aprobación de sus planes de estudios" [art. 2.2. d)], que en el caso de enseñanzas conducentes a la obtención de títulos de carácter oficial y validez en todo el territorio nacional deberá ajustarse a lo dispuesto en el Título VI de la LOU relativo a las enseñanzas y títulos, donde se remite al Gobierno la ordenación de las enseñanzas universitarias oficiales ${ }^{3 .}$

En consonancia con los principios consagrados en la LOU, el Real Decreto 1393/2007, de 29 de octubre, por el que se establece la ordenación de las enseñanzas universitarias oficiales ${ }^{4}$, profundiza en la concepción de la autonomía universitaria, dejando que sean las propias Universidades las que, de acuerdo con las reglas fijadas en el mismo, creen y propongan las enseñanzas y títulos que han de impartir y expedir, sin necesidad de sujetarse a un catálogo previo establecido por el Gobierno, como sucedía hasta ese momento 5 .

Por otra parte, como se advierte en el Preámbulo del propio Real Decreto, la nueva organización de las enseñanzas universitarias no se limita a un cambio estructural, sino que pretende además impulsar un cambio en las metodologías docentes, cuyo objetivo nuclear ha de ser el proceso de aprendizaje del estudiante. De ahí que, sin excluir el enfoque tradicional basado en contenidos y horas lectivas, los planes de estudios conducentes a la obtención de un título deben ahora centrarse en la adquisición de competencias por parte de los estudiantes, haciendo especial hincapié en los métodos de aprendizaje de dichas competencias y en los procedimientos para evaluar su adquisición.

\footnotetext{
${ }^{2}$ Sobre la autonomía de las Universidades, en general, y su regulación en la LOU, en particular, ver Souviron y Palencia (2002).

${ }^{3}$ Arts. 35.1 y 37 de la LOU, ambos en redacción dada por la Ley Orgánica 4/2007, de 12 de abril, por la que se modifica la Ley Orgánica 6/2001, de 21 de diciembre, de Universidades. Art. 35.1: "El Gobierno establecerá las directrices y las condiciones para la obtención de los títulos universitarios de carácter oficial y con validez en todo el territorio nacional..."; y art. 37: "Las enseñanzas universitarias se estructurarán en tres ciclos: Grado, Máster y Doctorado. La superación de tales enseñanzas dará derecho, en los términos que establezca el Gobierno, previo informe del Consejo de Universidades, a la obtención de los títulos oficiales correspondientes",

${ }^{4}$ BOE núm. 260, de 30 de octubre de 2007. El Real Decreto 1393/2007 ha sido modificado por el Real Decreto 861/2010, de 2 de julio (BOE núm. 161, de 3 de julio de 2010), si bien la modifícación no afecta a la regulación de la asignatura TFG.

${ }^{5}$ De acuerdo con el art. 34.1 y 2 de la LOU, en su redacción originaria, los diferentes títulos de carácter oficial y validez en todo el territorio nacional eran establecidos específicamente por el Gobierno de la Nación, integrándose en el Catálogo de Títulos Universitarios Oficiales, que también debía aprobar el Gobierno; como bien señalan Souviron y Palencia (2002) en la pág. 322, ello suponía que tales títulos oficiales constituían "un $<<$ numerus clausus $>>$, ampliable tan solo con el establecimiento por el Gobierno de un nuevo título oficial".
}

(cc) EY-NC-ND 2017, Universitat Politècnica de València 
En este marco conceptual, el art. 12 del citado Real Decreto 1393/2007, relativo a las directrices para el diseño de títulos de Graduado, establece en sus apdos $2^{\circ}, 3^{\circ}$ y $7^{\circ}$ las siguientes reglas básicas sobre el Trabajo de Fin de Grado:

-La obligatoriedad de que las enseñanzas de Grado concluyan con la elaboración y defensa de un TFG, lo cual significa que el TFG es una asignatura obligatoria que el alumno debe superar para poder obtener su título;

-La asignación al TFG de un mínimo de 6 créditos y un máximo de 30;

-El momento temporal en que debe realizarse el TFG, a saber, la fase final del plan de estudios: $\mathrm{y}$

-La necesidad de que el TFG esté orientado a la evaluación de competencias asociadas al título de que se trate.

En aplicación de tales preceptos, el Plan de Estudios del Grado en Criminología de la Universidad de Málaga establece la superación por el alumno de una asignatura de TFG de carácter obligatorio, con un contenido de 12 créditos ECTS, que se desarrolla en el segundo cuatrimestre del cuarto curso. Los estudiantes podrán matricularse en la misma siempre que cumplan los requisitos establecidos en la Memoria de Verificación del título, esto es, que haya superado el $70 \%$ de los créditos que conforman el plan de estudios.

El TFG consiste en un trabajo autónomo e individual que cada estudiante realizará bajo la orientación de un tutor, quien actuará como dinamizador y facilitador del proceso de aprendizaje (Ferrer, Carmona y Soria, 2012). Debe ser un trabajo original no presentado con anterioridad por el estudiante para superar otras materias en cualquiera de las Titulaciones a las que resulta de aplicación el presente Reglamento, o en otras titulaciones previamente cursadas en esta $\mathrm{u}$ otras universidades. Teniendo en cuenta las características mencionadas, podrán plantearse, en cuanto a su contenido, distintos tipos de TFG que permitan, a su vez, evaluar el grado de adquisición por parte de los estudiantes de las competencias definidas para esta materia en las respectivas Memorias de Verificación.

El contenido del TFG puede referirse a: trabajos empíricos o de campo; análisis y estudio acerca de la jurisprudencia de tribunales tanto nacionales como internacionales en relación a las materias establecidas por los Departamentos; estudios, informes o dictámenes originales vinculados a alguna/s de la materias desarrolladas en el Grado; cualquier otra investigación que se adecue a los contenidos y competencias que conforman la titulación de Graduado en Criminología.

La función de evaluación y calificación del TFG se atribuye en exclusiva a los Tribunales de Evaluación, mediante la aplicación de las rúbricas para la evaluación de competencias del Trabajo de Fin de Grado establecidas en el Reglamento del TFG de la Facultad de Derecho de la UMA (art. 11.1), las cuales se dividen en tres categorías: aspectos formales y estructura del TFG, expresión escrita y expresión oral y capacidad de síntesis del alumno en la exposición del trabajo, con una ponderación del $50 \%, 25 \%$ y $25 \%$, respectivamente (Castro, Cerezo y Benítez, 2015). 
Los criterios de valoración son los siguientes:

Aspectos formales y estructurales del TFG. (50\%)

El TFG cumple los requisitos formales de presentación.

El alumno ha acudido a las sesiones de trabajo con el tutor, incorporando sus sugerencias y comentarios y ha seguido el plan de trabajo establecido.

Originalidad.

Dificultad

Están definidas de forma clara las hipótesis y los objetivos del TFG.

Están identificadas las partes fundamentales del TFG.

Se exponen con claridad los resultados y se realiza una discusión de los mismos.

Las conclusiones del TFG responden a las hipótesis y objetivos planteados inicialmente.

Expresión escrita: $(25 \%)$

El alumno expresa, por escrito, de forma clara las ideas y objetivos, siguiendo las normas gramaticales y ortográficas.

El alumno utiliza apropiadamente terminología y vocabulario adecuados al tema.

El alumno cita adecuadamente el material empleado, utilizando bibliografía actualizada y apropiada al tema del TFG.

Expresión oral y capacidad de síntesis: $(25 \%)$

El alumno se expresa con claridad en la presentación oral del TFG.

El alumno muestra capacidad de síntesis en la presentación del trabajo realizado.

El alumno responde adecuadamente y de forma razonada a las preguntas que se le formulan.

De acuerdo con la normativa que acabamos de analizar, el TFG debe estar orientado a la adquisición tanto de las competencias generales del título de que se trate, en nuestro caso, el Grado en Criminología de la UMA, como de las competencias específicas asignadas a esta asignatura, recogidas ambas en la Memoria de Verificación del Grado en Criminología (arts. 12.7 del Real Decreto 1393/2007; 3.2 del Reglamento del TFG de la UMA; y 2.1 del Reglamento del TFG de la Facultad de Derecho de la UMA).

Si bien no es éste el lugar para detenernos en el estudio del concepto de competencia en el ámbito educativo, dada la importancia que la determinación de las 
competencias correspondientes a las titulaciones universitarias presenta en el diseño de los respectivos planes de estudio ${ }^{6,}$ nos parece oportuno ofrecer un breve esbozo del mismo.

La Comisión Europea define la competencia como "la capacidad demostrada de utilizar conocimientos y destrezas", considerando el conocimiento como el resultado de la asimilación de información que tiene lugar durante el proceso de aprendizaje y la destreza como la habilidad para aplicar conocimientos y utilizar técnicas a fin de completar tareas y resolver problemas (Feito, 2010). Por su parte, en el denominado Informe DeSeCo (Definición y Selección de Competencias), elaborado por la OCDE, se afirma que "la competencia es más que conocimientos y destrezas. Involucra la habilidad de enfrentar demandas complejas, apoyándose en y movilizando recursos psicosociales (incluyendo destrezas y actitudes) en un contexto en particular"7 Así, pues, la educación basada en competencias trata de formar a las personas no solo para que puedan participar en el mundo laboral, sino para que sean capaces de desarrollar un proyecto personal de vida (Feito, 2010).

Como puede apreciarse, el concepto de competencia educativa se presenta como un concepto complejo, lo que, unido a la falta de acuerdo entre los agentes llamados a intervenir en el contexto del EEES (el Ministerio de Educación, las Agencias para la calidad del sistema universitario -estatal y autonómicas-, y los expertos en pedagogía) en cuanto a su utilización en un sentido unívoco, ha podido coadyuvar a que el catálogo competencial establecido en los planes de estudio de nuestras titulaciones universitarias no presente en muchas ocasiones la formulación más adecuada (Bolivar, 2008).

Aclarados estos extremos, pasamos a enunciar las competencias generales y específicas $^{8}$ asociadas a la asignatura TFG en la Memoria de Verificación del Grado en Criminología de la UMA:

\footnotetext{
${ }^{6}$ A este respecto, Bolivar (2008), afirma que "el debate sobre el nuevo diseño de las titulaciones en términos de competencias no es sólo técnico, primariamente es político e ideológico: cuál debe ser la función de la Universidad en relación con la formación (formar para empleo o introducir en un ámbito cultural) y el tipo de profesionalidad requerida. El discurso de las "competencias" supone redefinir la profesionalidad como la regulación de un listado de competencias para la enseñanza, que los estudiantes han de adquirir para conseguir el título. En un plano general, lo que se discute es el mismo modelo de Universidad, que debe mediar entre modelo académico (Humboldt) y el profesionalizador (anglosajón o neoliberal). Una herencia de la educación liberal es que la enseñanza universitaria ha de proporcionar una formación de la inteligencia y el saber crítico, conjugada con un saber especializado profesionalizador".

${ }^{7}$ No obstante, como señala Bolivar (2008), conviene advertir que el Proyecto DeSeCo se dirige a la educación obligatoria, no a la universitaria.

${ }^{8}$ Como ponen de manifiesto Sánchez-Elvira, López y Fernández (2010), la necesidad de que la educación superior asuma como objetivo final la preparación de los ciudadanos para los nuevos retos del siglo XXI ha llevado a incorporar en los diseños de las titulaciones, junto a las competencias profesionales específicas, las denominadas competencias genéricas o transversales, entendidas como capacidades y destrezas que pueden ser utilizadas en muchas situaciones, no sólo en aquellas relacionadas con el área de estudio concreta, las cuales constituyen piezas clave de la nueva formación universitaria en su apuesta por potenciar la empleabilidad y el aprendizaje a lo largo de la vida. De este modo, el estudiante podrá desempeñarse de forma eficaz en un mercado laboral
} 


\section{-Competencias generales del Título (CG):}

CG 1.6. Utilizar herramientas propias del método científico para la planificación, diseño y ejecución de investigaciones básicas y aplicadas desde la etapa de reconocimiento hasta la evaluación de resultados y conclusiones.

CG 1.7. Realizar, evaluar y ejecutar proyectos e informes científico-técnicos relacionados con la criminalidad.

CG 1.8. Conocer y aplicar técnicas y procedimientos para la resolución de problemas y la toma de decisiones (desde una perspectiva sistémica que fomente la visión global del problema, su análisis y las interrelaciones existentes entre los mismos).

CG 1.9. Saber transmitir información, ideas, problemas y soluciones de carácter criminológico tanto a un público especializado como no especializado.

CG 1.10. Ser capaz de trabajar en equipo con otros profesionales en las diferentes vertientes de la actividad criminológica.

CG 1.12. Utilizar las Tecnologías de la Información y la Comunicación en la resolución de problemas y búsqueda de información en el ámbito de la Criminología.

-Competencias específicas (CE):

CE 2.7. Saber diseñar un proyecto de investigación empírico, aplicar técnicas de investigación cuantitativa y cualitativa, y analizar estadísticamente los datos.

CE 2.8. Saber difundir y exponer adecuadamente los resultados de una investigación en congresos académicos y a los medios de comunicación.

2.9. Saber elaborar e interpretar un informe criminológico.

2.10. Saber elaborar y evaluar programas y estrategias de prevención y/o intervención de la criminalidad.

CE 2.11. Conocer las técnicas de evaluación de riesgo de violencia y delincuencia y saber aplicarlas a casos concretos.

CE 2.19. Manejar los términos criminológicos en lengua inglesa.

\section{Objetivos}

El objetivo principal del presente trabajo es facilitar tanto a profesores como a alumnos la oportunidad de que los segundos vayan adquiriendo y reforzando durante la carrera las competencias que con posterioridad se van a exigir en la asignatura de trabajo fin de grado (en adelante TFG) del Grado de Criminología de la Universidad de Málaga.

dinámico, adaptarse de forma flexible a los rápidos cambios y demandas sociales y, en definitiva, constituirse en agente activo en la construcción de la sociedad del conocimiento.

(cc) EY-NC-ND 2017, Universitat Politècnica de València 
Desde que se comenzó a impartir la asignatura de TFG venimos observando una importante carencia en los alumnos que cursan esta asignatura en el último año de carrera. Los tutores que dirigimos estos trabajos nos hemos encontrado con alumnos que redactan con mucha dificultad, incluso con faltas de ortografía, que no saben realizar citas bibliográficas o incluso ignoran una serie de normas básicas estructurales respecto a la elaboración de un trabajo. Ello produce una situación de alta frustración tanto en los alumnos como en sus profesores.

Entendemos que este problema se pueda resolver a través de una herramienta docente que sea capaz de integrar las competencias que deben adquirir los estudiantes y, al mismo tiempo, sea una experiencia de trabajo coordinado para los profesores.

\section{Desarrollo de la inovación. Plan de trabajo por competencias y semestre: una nueva herramienta docente.}

La metodología que se va a seguir a fin de conseguir nuestro objetivo consiste en fijar durante los tres primeros cursos de la titulación del grado de Criminología un plan de trabajo determinado, de tal forma que durante un curso y un semestre se trabajen de manera coordinada por los profesores que imparten docencia en ese curso y semestre una competencia específica del Grado.

El profesor de una asignatura elegirá, en función de la competencia asignada a trabajar en ese curso y semestre, qué actividades quiere poner en práctica con los alumnos de su grupo. Para ello seleccionará aquellas que les parezca oportunas de un amplio cuadro que se ofertará, a modo de sugerencia. A la vez que el alumno va adquiriendo los conocimientos propios y exigidos de la materia, va trabajando conscientemente sobre las competencias que con posterioridad le serán valoradas en la asignatura de Trabajo Fin de Grado. En cada curso académico se van añadiendo competencias de modo que el alumnado de cuarto curso que se matricule del Trabajo Fin de Grado habrá podido ir aprendiendo tanto una metodología como el uso de las herramientas oportunas para superar satisfactoriamente la asignatura.

Para realizar este plan de trabajo será imprescindible la colaboración del mayor número posible de profesores, de tal modo que para cada curso y semestre se creen equipos docentes que sean capaces de coordinarse de cara a lograr que el estudiante adquiera las correspondientes competencias. Ello significa que a los alumnos de un curso, semestre y grupo se le deben proponer actividades no reiterativas. Aconsejamos igualmente que estas pequeñas actividades se incardinen en la evaluación docente de las correspondientes asignaturas, de tal forma que su realización sea prácticamente preceptiva. Como apoyo al sistema descrito se realizarán seminarios y sesiones informativas y de trabajo con los profesores de cada curso y semestre para incrementar la coordinación en la práctica docente. 


\section{Resultados preliminares}

La lista de habilidades a trabajar en cada competencia y propuesta de actividades en el Grado de Criminología son las siguientes:

\section{Primer curso primer y segundo semestre.}

Objetivo: Uso de las Tecnologías de la Información y la Comunicación en la búsqueda de información en el ámbito de la Criminología:

a) Realización del curso que se oferta en biblioteca: Manejar bibliografía adecuada e indicar correctamente las referencias bibliográficas empleadas tanto a lo largo del texto del trabajo como en el apartado de bibliografía.

b) Búsqueda de fuentes de información estadística o de carácter secundario.

c) Búsqueda de Bibliografía: Lectura y recensión de artículos doctrinales para cuya localización se facilitará solamente la referencia de la Revista científica.

\section{Segundo curso primer semestre}

Objetivo: Utilización de herramientas propias del método científico para la planificación y diseño de investigaciones empíricas. Se persigue la adquisición de las siguientes habilidades:

a) Diseño de un proyecto de investigación empírico.

b) Elaboración del marco teórico

c) Elaboración de objetivos e hipótesis de estudio

d) Aplicación de técnicas de investigación cuantitativa y/o cualitativa.

\section{Segundo curso segundo semestre}

Objetivo: Realización de proyectos e informes científico-técnicos relacionados con la criminalidad. Se persigue que se trabajen las siguientes habilidades:

a) Orden expositivo escrito.

b) Cumplimiento del formato establecido.

c) Expresión escrita ortográfica y gramatical.

d) Idoneidad de las referencias usadas.

e) Trabajo en equipo

f) Análisis cuantitativo y/o cualitativo de los datos recogidos

g) Revisión crítica y aplicación práctica a partir de las conclusiones obtenidas.

\section{Tercer Curso primer semestre:}

Objetivo: Exposiciones orales de temas de programación docente o de trabajos de investigación. Se persigue que se trabajen las siguientes habilidades:

a) Orden expositivo oral. 
b) Cumplimiento de los tiempos establecidos.

c) Adecuada modulación de la voz.

d) Uso del lenguaje corporal y oral.

e) Claridad, ritmo y organización de la exposición.

f) Respuesta adecuada y pertinente argumentación a las preguntas y comentarios del profesor y los compañeros.

\section{Tercer Curso segundo semestre:}

Objetivo: Análisis crítico y toma de decisiones. Se persigue que se trabajen las siguientes habilidades:

a) Grado de coherencia en el razonamiento.

b) Justificación del desarrollo y conclusiones.

c) Resolución de problemas desde distintos ámbitos de la Criminología.

d) Toma de decisiones desde una perspectiva sistémica que fomente la visión global del problema, su análisis y las interrelaciones existentes entre los mismos.

Para los profesores-tutores de trabajos fin de grado se elaborará y facilitará un diario de evaluación para los alumnos a los que tutorizan. Ese diario estará formado por una serie de documentos que se ofrecerán como herramienta que facilite tener un control documentado de la evaluación de las competencias, del ritmo y resultado del trabajo del estudiante a lo largo de la elaboración del Trabajo fin de Grado y en el que se haga constar la dedicación real a cada alumno y trabajo para que en la medida de los posible se refleje la necesidad de reforma de la dedicación a estos trabajos que reconoce el POD.

A modo de ejemplo contará con los siguientes documentos:

a) Acuerdo suscrito entre alumno y profesor por el cual el alumno se compromete a asistir con aprovechamiento a las tutorías que determine el profesor tutor y a cumplir con el plan de trabajo que fije el tutor.

b) Hoja de control de entregas periódicas del trabajo al tutor, con observaciones de si el estudiante ha incorporado sus sugerencias y comentarios y ha seguido el plan de trabajo establecido.

c) Hoja de verificación de definición de hipótesis, objetivos y conclusiones del trabajo.

d) Hoja de verificación de metodología y partes del trabajo.

e) Hoja de verificación de terminología y uso adecuado de vocabulario.

f) Hoja de verificación de uso de Bibliografía apropiada y actualizada.

g) Hoja de verificación del tiempo dedicado a cada alumno.

\section{Conclusiones}

La asignatura de Trabajo Fin de Grado en las nuevas titulaciones españolas está suponiendo un cambio docente significativo. Muchos asimilan esta asignatura a los 
anteriores proyectos fin de carrera, pero lo cierto es que nos encontramos ante una situación diferente y novedosa. En el Grado de Criminología que se imparte en la Facultad de Derecho de la Universidad de Málaga llevamos un tiempo trabajando para conseguir dotar de contenido y coherencia a esta híbrida asignatura.

La tarea no es fácil, si tenemos en cuenta que se trata de una asignatura como otra cualquiera del plan de estudios pero con unas características muy específicas, ya que son un gran número de profesores los implicados en la misma. Por otro lado, nos encontramos con la especificidad de que para superarla es necesaria la elaboración de un trabajo de investigación, a través del cual el alumno tiene que demostrar que ha alcanzado todas las competencias que ha ido adquiriendo durante la carrera.

En el presente artículo hemos intentado dar solución a todas las dificultades que atraviesa esta asignatura de Trabajo Fin de Grado. Principalmente, hemos querido plasmar la idea, basada en nuestra corta experiencia como docentes de dicha asignatura, de que los estudiantes se enfrentan a la realización de un TFG sin los conocimientos suficientes acerca de su elaboración y desarrollo. Ello es debido a que durante la carrera no han ido adquiriendo de forma paulatina las competencias necesarias para alcanzar el nivel que se les exige en la etapa final de sus estudios universitarios.

Para solventar esta dificultad, hemos ideado una herramienta de trabajo que se basa en la coordinación de los profesores de cara a proponer diferentes actividades durante el desarrollo de las asignaturas curriculares y de esta forma preparar adecuadamente al alumno que se enfrenta a la elaboración de un TFG.

\section{Referencias}

Bolivar Botia, A. (2008). "El discurso de las competencias en España: educación básica y educación superior", en Red U. Revista de Docencia Universitaria, 2, p.1-23.

Castro, M.P., Cerezo, A.I. y BeníteZ, M.J. (2015). "Diseño de un procedimiento objetivo para la evaluación y calificación de la asignatura de Trabajo Fin de Grado en el Grado de criminología de la Universidad de Málaga", en Revista Jurídica de Investigación e Innovación Educativa (REIJE), 12, p.1-19.

Feito Alonso, R. (2010). "De las competencias básicas al currículum integrado", en Qurriculum: Revista de teoría, investigación y práctica educativa, 23.

Ferrer, V., CARmona, M. y Soria, V. (2012). El Trabajo de Fin de Grado. Guía para estudiantes, docentes y agentes colaboradores. Nueva York: MacGraw-Hill.

OCDE, Informe DeSeCo: La definición y selección de competencias clave. Resumen Ejecutivo.

SÁNCHEZ-ELVIRA, A., LÓPEZ-GONZÁLEZ M.A. Y FernANDEZ-SÁNCHEZ, M.V. (2010). "Análisis de las competencias genéricas en los nuevos títulos de grado del EEES en las Universidades españolas", en Red U. Revista de Docencia Universitaria, vol 8, 1, p. 1-39.

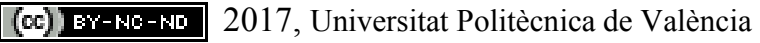


SOUVIRON Morenilla, J.M. y PALENCIA HERREJón, F. (2002). La nueva regulación de las Universidades. Comentarios y Análisis sistemático de la Ley Orgánica 6/2001, de 21 de diciembre, de Universidades. Granada: Comares.

\section{Legislación y normas}

España. Ley Orgánica 6/2001, de 21 de diciembre, de Universidades. BOE, 14 de diciembre de 2001, núm. 307, p. 49400 - 49425.

España. Ley Orgánica 4/2007, de 12 de abril, por la que se modifica la Ley Orgánica 6/2001,

de 21 de diciembre, de Universidades. BOE, de 13 de abril de 2007, núm. 89, p. 16241 -

16260.

España. Real Decreto 1393/2007, de 29 de octubre, por el que se establece la ordenación de las enseñanzas universitarias oficiales. $B O E$, de 30 de octubre de 2007, núm. 260, p. 44037 $-44048$.

España. Real Decreto 861/2010, de 2 de julio, por el que se modifica el Real Decreto 1393/2007, de 29 de octubre, por el que se establece la ordenación de las enseñanzas universitarias oficiales. $B O E$, de 3 de julio de 2010, núm. 161, p. 58454 - 58468. 